\title{
Coping Mechanisms of Senior High School Students with Sleep Deprivation: A Phenomenological Study
}

\author{
Leslie Parantar a,f, Jessel Reforzado b,f, Gabrielle Costuna ${ }^{\mathrm{c}, \mathrm{f}}$, Felvieh Llurag d,f, \\ Rolando De Veyra ${ }^{\mathrm{e}, \mathrm{f}}$ \\ a leslieannptr5@gmail.com \\ b jesseljaner@gmail.com \\ c gabreilcostuna@gmail.com \\ d Felviehl@gmail.com \\ esouleater14344@gmail.com \\ ${ }^{\mathrm{f}}$ Leyte Normal University - Integrated Laboratory School, Tacloban City 6500, Phiippines
}

\begin{abstract}
Sleep deprivation is so prevalent nowadays that anybody, regardless of age, can suffer from it. Teenagers are more vulnerable in terms of academic performance, emotional and mental challenges. In this study, researchers look primarily at the coping strategies of senior high school students who are sleep deprived. The researchers first chose the Senior High School Students of Leyte Normal University-Integrated Laboratory School as research participants. The researchers subsequently constructed the researcher-made questionnaires, which were first approved by the Research Advisor. The questionnaires were then given to the participants. The researchers used Colaizzi's (1978) data analysis technique to evaluate, define, and portray the experiences of sleep-deprived students, as well as to reveal developing trends.

Despite having experienced varying degrees of sleep deprivation, the subjects (Students) exhibited comparable coping techniques. The most common is the consumption of any beverage, whether caffeinated, milk, hot tea, or even cold water. Some participants keep themselves occupied by listening to music, browsing social media platforms, and engaging in physical activities such as exercising, taking a bath, and actively participating in class discussions. These are the typical coping techniques that the students use to avoid sleepiness. And it appears that the students did well in combating sleep deprivation; so, we can claim that sleep deprivation is something that we can regulate via or with effort. As a result, it is essential that students create an appropriate itinerary to assist them maintain a regular balance between their personal life, academics, and relaxation time.
\end{abstract}

Keywords: Sleep deprivation; Coping mechanism; Phenomenological study

\section{Introduction}

Sleep is a natural occurrence that all individuals require in order to restore their energies. Sleeping takes up one-third of our lives so a good night's sleep is essential for improving cognitive abilities, particularly memory retention (Cureus, 2019). Failure to get a decent night's sleep can have a variety of effects to an individual. 
According to Merriam-Webster dictionary, sleep deprivation is the act of not getting enough sleep. A drowsy tired person is more prone to accidents, has poor judgment, and is more likely to make blunders and unwise decisions (BetterHealth Channel, 2014). As for teenagers, they are most likely to be affected in terms of academic performance, emotional, and mental difficulties.

Sleep deprivation has no bounds. Anyone, regardless of age, can be a victim. It has gotten so bad that the American Academy of Pediatrics (2014) declared the issue of weary students to be a public health epidemic (Richter, 2015). A consensus recommended, to improve health, teens aged 13 to 18 should sleep 8 to 10 hours every 24 hours on a regular basis (Paruthi, et al., 2016). However, it is frequently not reached due to a variety of circumstances that vary based on the students' surroundings. According to Richter (2015), students' biology has clashed with social and cultural forces, as well as the advent of technology, preventing them from obtaining adequate sleep.

With this, Richter (2015) noted that sleep deprivation among students are more likely to suffer from a variety of negative effects, such as an inability to focus, low grades, drowsy driving accidents, anxiety, despair, suicidal thoughts, and even suicidal attempts. The problem of sleep deprivation among the students should be taken seriously because it can directly affect the overall performance.

Students should be aware of the coping techniques connected with sleep loss in order to avoid these side effects. Furthermore, the school must also assist in avoiding this problem from arising among its students. Thus, the researchers seek to investigate effective coping methods among the senior high school students of Leyte Normal University - Integrated Laboratory School in this study.

\subsection{Research Questions}

The study's goal is to discover coping strategies in sleep deprivation among LNUILS senior high school students. It will specifically seek answers to the following questions:

1. Why do senior high school students experience sleep deprivation?

2. How does sleep deprivation affect one's role as a student and as a member of the family?

3. What are the coping mechanism techniques that senior high school students do?

\subsection{Definition of Terms}

The words mentioned here are provided to offer readers a shared understanding of how they were used in the study.

Sleep deprivation. Refers to a situation induced by insufficient sleep quantity or quality, which can include both intentional and unintentional sleeplessness as well as circadian rhythm sleep disorders. ("Sleep Deprivation", 2014).

Coping mechanism. Defined as ideas and behaviors used to cope with internal and external stressful events (Algorani \& Gupta, 2021). 
Phenomenological study. Examines what people went through and focuses on their reactions to a situation ("Qualitative Research Methods", 2020).

\subsection{Theoretical Framework}

This study is founded on the Coping Theory, Lazarus, R. \& Folkman, S. (1984) defined coping as: constantly changing cognitive and behavioral efforts to manage specific external and internal demands that are appraised as taxing or exceeding the resources of the person. Coping involves spending mental energy in a way that can reduce stress. Whether conscious or subconscious, the ultimate goal of all coping mechanism is to solve a problem and return homeostasis. The coping theory is a vast area of study that is classified into two independent parameters: Focus-oriented theories (trait and state) and Approach-oriented theories (microanalytic and macro-analytic). The focus-oriented state and trait theories of coping recognize a person's internal resources and mental capacities for evaluating how well he can adapt to a situation. On the other hand, the approach-oriented micro and macro analytic coping theories revolve around how concrete or abstract the coping mechanisms are (Carver, Scheier, \& Weintraub, 1989). Psychologists agree that coping mechanisms vary from person to person and from time to time. No two people would use the same strategies to get over a situation. Even the same individual can use two completely different coping strategies to adapt to similar stressors at different points in life. The eight functions by Lazarus forms the base of active emotional coping and lies at the core of our behavior while experiencing stress. Lazarus and Folkman coined the concept of cognitive appraisal and reappraisal. According to their theory, stress coping implies an intricate process of thinking and assigning meaning to it. They explained the coping mechanism by the stress cycle where an individual's perception of the stressful situation decides how he would cope with it (Anshel, 1996; Anshel \& Weinberg, 1999; Roth \& Cohen, 1986).

There are several methods for dealing with stress. Seeking aid from supportive individuals, such as a counselor or friend, is one of the positive coping methods. Meditation, writing, and exercise are also effective coping mechanisms.

A negative coping strategy is when a person assaults others and makes them feel uncomfortable. Alternatively, we might avoid the person, location, or object that causes us stress. Some people choose to be defensive or even injure themselves.

\section{Review of Related Literature}

During sleep, brain activity varies, rising and decreasing during the many sleep phases that make up the sleep cycle which includes 4 stages: one is for rapid eye movement (REM) sleep, while the other three are for non-REM (NREM) sleep (Suni, 2020). In REM sleep, it is thought to be necessary for cognitive activities such as memory, learning, and creativity (Suni, 2020). Each stage is important for brain health because it allows activity in different regions of the brain to increase or decrease, allowing for improved thinking, learning, and remembering (Suni, 2020). Failure to get adequate deep sleep and REM sleep may explain some of the significant effects of sleep deprivation on thinking, emotions, and physical health (Suni, 2020). 
Sleep deprivation is a condition that occurs as a result of a lack of sleep. A teenager needs roughly nine hours of sleep every night (Noland et al., 2009). Sleep deprivation can occur if a teenager sleeps for less than nine hours (Bergin et al., 2009).

Academic Performance refers to the knowledge that has been established by the students during their academic years. Educational institutions learn whether or not they have achieved their goals with the help of monitoring how students perform within a particular period.

According to a study done by Noland and his colleagues in the Midwest, most high school students get insufficient sleep (less than 9 hours) on most school evenings of the week, with 10\% reporting less than 6 hours of sleep each week night (Noland et al, 2009). Insufficient sleep can cause changes in the human body and mind, which might have an impact on the student's life (Axelrod et al, 2010). Sleep deprivation is referred to as "pulling an all-nighter" among students, which means staying up for 24 hours or longer (Hershner \& Chervin, 2014). Not having enough sleep can gradually become more serious as time goes by which generates to poor concentration and to having experiences of sleepiness even during daytime. According to Patrick et al. (2017), sleep deprivation has been correlated with poor academic performance and physical condition. Studies investigating the effects of different sleep-wake schedules on academic achievement showed that bad sleep quality and quantity are related to decreased learning ability and daytime functioning (Alfonsi, 2020). It also has a crucial impact on the health, wellbeing, and academic performance to adolescents (Gaultney, 2010; Haile et al., 2017).

Although it is unclear how sleep aids learning, data from a research by Yang et al. (2014) suggest that sleep plays a critical role in boosting learning-dependent synapse creation and maintenance on specific dendritic branches, which aid memory storage.

According to Yang et al. (2014), "sleep increases the development of postsynaptic dendritic spines on a subset of branches of individual layer $\mathrm{V}$ pyramidal neurons following motor learning. When numerous tasks are learnt, new spines are generated on distinct sets of dendritic branches in response to diverse learning tasks, and they are safeguarded from being destroyed. Neurons that were engaged during motor task learning are reactivated during subsequent non-rapid eye movement sleep, and blocking this neuronal reactivation inhibits the development of branch-specific spines." Which led to Zeek et al. (2014) to hypothesize that greater sleep duration will lead to higher academic performance based on the scientific basis linked to the influence of sleep on cognitive.

Sleep deprivation affects neuronal activity in the frontal and parietal cortices, which are important for working memory, and decreases general attention and mnemonic ability (Frenda \& Fenn, 2016). Under situations of sleep loss, poor task performance is generally associated by decreased activation in task-relevant regions (Frenda \& Fenn, 2016). Sleep deprivation for 24 hours dramatically increase stress hormone levels, and decrease attention and working memory (Joo et al., 2011). With this, the effect of sleep deprivation on cognitive performance has also been recorded as a correlation between sleep quality and the average grade of students. On top of that, sleep deprivation has been shown to have a damaging effect on particular aspects of working memory, such as filtering productivity, whilst test scores show reduction. It was also showed in a study conducted to college students, that sleep deprived participants performed considerably worse than nondeprived ones on the cognitive task (Pilcher \& Walters, 2010). These propose that sleep deprivation may have a limited effect on cognitive ability in students (Patrick et al., 2017). 
In relation to that, sleep deprivation has also shown some consequences in critical skills negatively affecting the leadership skills of a student. A result from a study conducted by Chen \& Chen (2019) showed that chronic sleep deprivation is negatively associated with lower scores in leadership skills and need for cognition from both of their models (random-effects and fixed-effects models).

Effects vary on the consistency of not getting enough sleep. Pulling an all-nighter may result to short-term effects on the brain and cognition while individuals with persistent sleep disorders may have trouble with dayto-day activities (Suni, 2020). Over the long term, poor sleep may increase the risk of cognitive decline and possibly dementia (Suni, 2020). Truly, the impact of sleep deprivation is really alerting, that sleep deprived people often say that they feel "foggy". With the reasons as follows: (1) slows down the cognitive process, (2) impaired memory, and (3) makes learning difficult (Peri, 2021).

Lack of sleep may also affect the mental health of individuals. It can cause mood swings such as irritation and rage, as well as a reduction in stress tolerance (Peri, 2021). The rise in serum cortisol levels associated with sleep loss has also been linked to mania disorder (Song et al., 2015). Another mental health problem that is linked to lack of sleep is depression. Both are so tightly linked that sleep experts can't tell which originated first but it is ideal to say that both are harmful to humans. In a study by Roberts \& Duong (2014) stated that reduced sleep quality raises the likelihood of severe depression, which raises the risk of sleep deprivation. In other words, sleeping problems can lead to changes in mental health, while mental health issues can exacerbate sleep issues (Cherry, 2020).

Coping is defined as activity that helps the people from being psychologically injured by negative social experiences, and it is essential because it mediates the influence that societies have on its members (Pearlin \& Schooler, 2016). In the case of sleep deprivation, a study showed that there were positive and negative coping strategies that the participants did. The coping mechanisms linked with improved sleep quality were napping and changing sleep patterns (Yang et al., 2010). Subjects who reported doing a sleep-promoting activity, disregarding their sleep issues entirely, or failing to find a strategy to cope with their sleep problems reported worse sleep quality (Yang et al., 2010).

In a study conducted by Nitin et al., (2013), they have concluded that coping methods also have an impact on residents by modifying how they perceive and react to stressful events, as well as affecting their sleep quality.

At the end of each day, sleep has a vital restorative role in 'recharging' the brain. Maintaining a consistent sleep-wake cycle allows the body's natural rhythm to be reset every day, which improves brain function and mental wellness (Bijlani, 2021). Better cognitive performance is also linked to greater sleep, and vice versa (Mantua \& Simonelli, 2019), improving productivity and overall quality of life (Suni, 2020). In order to obtain that, one must have a sleep hygiene that entails a sleeping environment as well as daily habits that encourage regular, uninterrupted sleep (Suni, 2020). Having a consistent sleep schedule, a pleasant and distraction-free bedroom, a soothing pre-bed ritual, and developing healthy behaviors during the day may all help you achieve optimal sleep hygiene (Suni, 2020). 


\section{Research Design And Methodology}

\subsection{Research Participants}

The participants of this study are the Senior High School Students of Leyte Normal University-Integrated Laboratory School who are currently enrolled this school year 2020-2021 and are identified as individuals experiencing sleep deprivation. They are labeled as P1, P2, P3, P4, P5, P6, P7, P8, P9, P10, P11. The reason for choosing the LNU-ILS senior high students as participants includes how it would be beneficial for both current and incoming senior high school students to raise their awareness about sleep deprivation; choosing senior high school students as our participants would make it easier to communicate with them considering the limited face-to-face contact we need to execute and implement as of the moment; the topic would not be complex for the reason that the topic is very relatable. Hence, it would be much easier to approach the topic as it is close to those particular students struggling academically due to being sleep deprived; the topic will possibly have a lot of respondents.

\subsection{Research Data Gathering Procedure}

\section{Step 1: Formulating of letters and questionnaires}

The researchers first devised a researcher-made open-ended and close-ended survey queries and interview questions.

\section{Step 2: Preparation for the collection of data}

Researchers then prepared the items required for the actual data collection. We first seek for an approval to our research advisor of our questionnaires. Once approved, questionnaires were double-checked and edited. The researchers created Google forms with questionnaires in them.

\section{Step 3: Actual gathering of data}

Following the planning, researchers began collecting data by connecting with each participant via Messenger. The participants first received a letter of consent outlining the reasons for the data collection, telling them that their identities and answers will be kept private, and requesting their permission to participate in the research by answering the survey questions. When participants chose to participate in the analysis, Google Form survey questionnaires were sent to them. After the participants answered the questionnaires, selected individual interviews were taken to collect further data. Data would be more valid and dependable this way.

\subsection{Data Analysis Method}

Colaizzi's (1978) method of data analysis is a rigorous and robust qualitative method that the researchers used to find, understand, describe and depict the experiences of students as they experience sleep deprivation, as well as reveal emergent themes. 


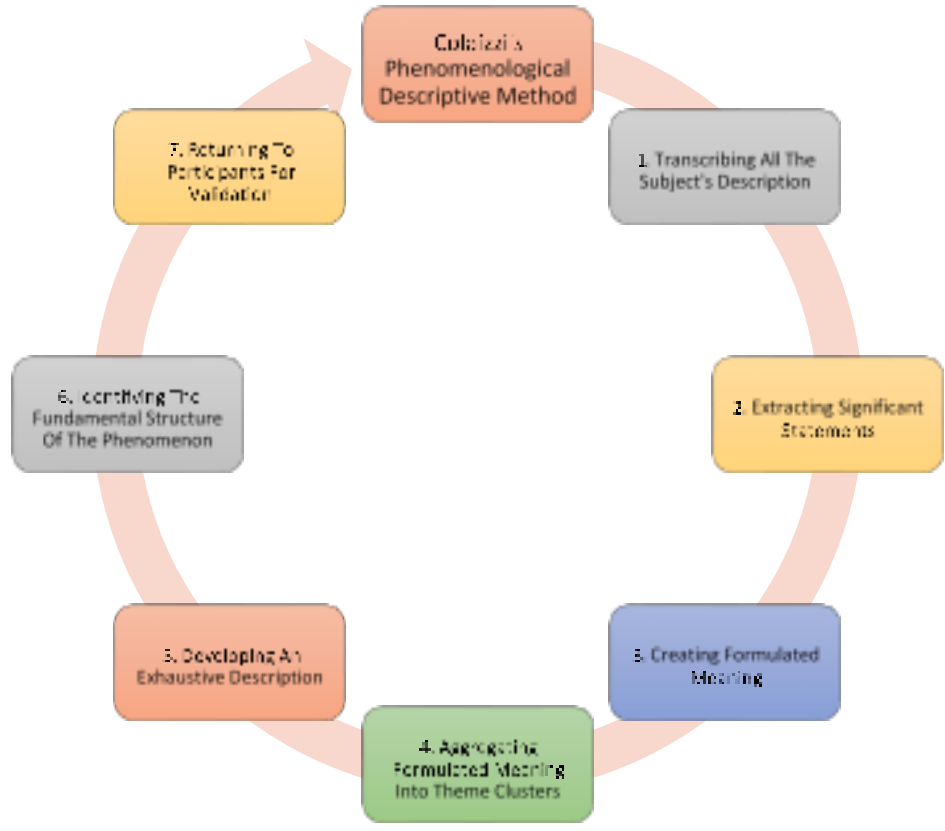

Fig. 1. Procedures of Colaizzi’s phenomenological descriptive method (Colaizzi, 1978)

\section{Results and Discussion}

The researchers initially transcribed the audio-recorded interviews and placed them in a single folder alongside the survey responses. The researchers were able to select and categorize the relevant material on the phenomenological inquiry of coping strategies of senior high school students suffering sleep deprivation after going through the responses from the survey questionnaire and audio-recorded interviews. The six major themes are as follows:

Theme 1: "With the help of coffee or any kind of energy drink.": DRINKING ENERGY BOOST

\section{BEVERAGES}

Theme 2: "I play exciting songs.": LISTENING TO MUSIC

Theme 3: "I play games so I can stay awake": RESORTING TO TECHNOLOGY

Theme 4: "I take a bath or wash my face as it kind of refreshes my mood and overall well-being. If I get sleepy, I'm going to get up from my bed and start exercising just to feel awake. ": RESORTING TO PHYSICAL ACTIVITIES

Theme 5: "I like to recite a lot because it keeps me engage in the class and it keeps me awake."

\section{: ENGAGING ACTIVELY TO SCHOOL TASKS}

Theme 6: "I do my daily routine like household chores.": DOING RESPONSIBILITIES

Theme 1: Drinking Energy Boost Beverages

Most of the participants stated that in order to stay awake, they either consume coffee, milk, water, or any hot drinks.

Participants stated:

"I drink coffee so I could be alert." (P2) 
"By drinking coffee" (P3)

"With the help of coffee or any kind of energy drink." (P11) "Coffee" (P9)

Theme 2: Listening to Music

Participants resort to listening to music particularly exciting ones to help them stay alert. Researchers think that the mix of tones that rise and fall in a song's melody "encourage arousal inside our system, which may contribute to enhanced alertness," according to Cory Stieg (2020).

Participant stated:

"Music also works" (P6)

"I play exciting songs." (P8)

Theme 3: Resorting to Technology

Two (2) of the participants rely on the use of technology as their mean of staying awake during the day. It helps them be entertained which lower the possibility of feeling drowsy. This includes playing mobile games and using social media.

Participant stated:

"I play games so I can stay awake" (P8)

"Playing games, or chatting with friends" (P4)

Theme 4: Resorting to Physical Activities

Doing physical activities may help in preventing the participants to think that they are sleepy. It does not only help them to keep themselves awake but it can also relieve stress from both online and offline tasks.

Participant stated:

"I take a bath or wash my face as it kind of refreshes my mood and overall wellbeing. If I get sleepy, I'm going to get up from my bed and start exercising just to feel awake." (P2)

"Making myself busy by doing a lots of activities such as exercising and biking" (P7)

"Eating, slapping myself, doing aerobic exercise" (P5)

Theme 5: Engaging Actively to School Tasks

This coping mechanism focus on students' active engagement on class participation. This makes the participants challenge their ideas on answering class recitation instead of falling asleep while synchronous classes.

Participants stated:

Mahilig ako mag participate ha class para diri ako mangaturog. (P1)

"I like to recite a lot because it keeps me engage in the class and it keeps me awake." (P2)

Theme 6: Doing responsibilities

Helping out with the household responsibilities has been a big help for the participants to be able to manage their personal responsibilities while keeping themselves awake.

Participants stated:

"Paghimo hin mga chores." (P3)

"Doing chores, pagbantay hit ak mga patud, ngan summer job." (P10) 


\section{Conclusion}

10 out of $10(100 \%)$ participants were reportedly experiencing sleep deprivation. Majority of the participants pointed out social media and online gaming as their main reason as to why they are sleep deprived, some of the students experience mental disorders such as anxiety, insomnia, and stress that give rise to the difficulty of being sleepy. Several students prefer doing their activities late at night and considers themselves nocturnal or a night owl since they are more active at night than daylight.

Based on a qualitative analysis of Sleep deprivation; Its effects on one's role as a student and as a member of the family, it can be concluded that sleep deprivation significantly affects one's behavior concerning their relationship with the people around them, most of the time a sleep deprived person tends to be stubborn and easily gets irritated even by the simplest things. Lack of sleep also does affect the behavioral approach of an individual towards their respective chores, assignments, and activities, this considerably affects their focus towards a certain task that has to be done as soon as possible.

Despite of experiencing different levels of sleep deprivation, the participants (Students) had shared similar coping mechanisms. Most common among all is the consumption of any drink it may be caffeinated, milk, hot tea, or even cold water. Some of the participants tend to keep themselves busy by resorting to either technology such as listening to music, scrolling through any social media platforms and resorting to physical activities, exercising, taking a bath, and active engagement in class discussions for instance. These are the usual coping mechanisms that the students utilized to prevent drowsiness, and it seems that the students had quite a good run on battling sleep deprivation, we can therefore say sleep deprivation is something that we can control through or with effort.

\section{Recommendation}

As the researchers finished gathering all the needed data for the study, they were able to come up with some recommendations. All of these were formulated from the results they arrived in. Looking at the results, it was summed up that the LNU-ILS senior high school students in both the STEM and HUMSS Strand had different ways to cope up with sleep deprivation. However, the researchers would also like to recommend the following:

- The participants mentioned that they were not able to have enough sleep because of stress and school works. To be able to cope with the lack of sleep, the researchers recommend that it is better to balance and come up with a time intended only for school and a time intended to relax.

- The participants mentioned that they frequently play games and use the social media especially during nighttime. The researchers recommend minimizing the use of gadgets because it minimizes the production of melatonin, which makes a person sleepy.

- The researchers recommend sleeping in a consistent sleep-schedule in order to find their own proper body clock.

\section{References}

Among teens, sleep deprivation on epidemic. Stanford Medicine. Retrieved June 21, 2021 from https://med.stanford.edu/news/allnews/2015/10/among-teens-sleep-deprivation-anepidemic.html 
Alfonsi, V. (2020). Later School Start Time: The Impact of Sleep on Academic Performance and Health in the Adolescent Population. MDPI. https://www.mdpi.com/1660-4601/17/7/2574

Algorani EB, Gupta V. Coping Mechanisms. [Updated 2021 May 3]. In: StatPearls [Internet]. Treasure Island (FL): StatPearls Publishing; 2021 Jan-. Available from: https://www.ncbi.nlm.nih.gov/books/NBK559031/

Axelrod, J. (2013). "Sleep Deprivation may Cause a Student Immense Limitation and Frustration." https://www.chagrinschools.org/Downloads/researcfhSleep\%20deprivation.pdf

Blame tired brain cells for mental lapses after poor sleep: Study reveals sleep deprivation disrupts brain-cell communication. (n.d.). ScienceDaily. https://www.sciencedaily.com/releases/2017/11/171106112312.htm

Bergin, Christi A., and David A. Bergin. "Sleep: The E-Z, The answer to low achievement might just be a good night's sleep." Dec. 2009. File last modified on Jan. 2010. PDF file.

https://kipdf.com/sleep-deprivation-may-cause-a-student-immense-limitation-and frustration_5ae2bc587f8b9a380b8b462a.html

Causes and consequences of sleepiness among college students. (n.d.). PubMed Central (PMC). https://www.ncbi.nlm.nih.gov/pmc/articles/PMC4075951/

Chen, W. L., \& Chen, J. H. (2019). Sleep deprivation and the development of leadership and need for cognition during the college years. Journal of adolescence, 73, 95-99. https://pubmed.ncbi.nlm.nih.gov/31048109/

Chowdhury, R. B. M. A. (2021, May 25). What is Coping Theory? PositivePsychology.Com. https://positivepsychology.com/copingtheory/?fbclid=IwAR2ozzvPpzKHD8NeP0cAIHKEyFe31J7T2TJZTQAjAA13eEow7ilhB_C4Wvk

Coping with Sleep Disturbances Among Young Adults: A Survey of First-Year College Students in Taiwan. (n.d.). Taylor \& Francis. Retrieved August 1, 2021, from https://www.tandfonline.com/doi/abs/10.1080/08964280309596066

Effects of sleep deprivation on cognitive and physical performance in university students. (2017). PubMed Central (PMC). https://www.ncbi.nlm.nih.gov/pmc/articles/PMC5489575/

Effects of sleep deprivation on serum cortisol level and mental health in servicemen. (2015, June 1). ScienceDirect. https://www.sciencedirect.com/science/article/abs/pii/S0167876015001567

Frenda, S. J., \& Fenn, K. M. (2016). Sleep less, think worse: the effect of sleep deprivation on working memory. Journal of Applied Research in Memory and Cognition, 5(4), 463-469. https://psycnet.apa.org/record/2016-61773-013

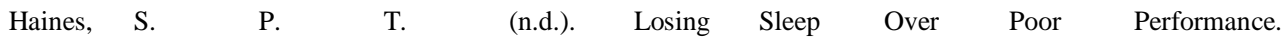
https://edtheory.blogspot.com/2015/09/losing-sleep-over-poorperformance.html?fbclid=IwAR001tR0QbmLFMJJrb6c5xKelrav0k0vZx1CwWR5njkOM4dLsWn7ceKLm8

How poor sleep affects your mental health. (n.d.). Priory Group. https://www.priorygroup.com/blog/how-poor-sleep-affects-your-mentalhealth?fbclid=IwAR2n_b6BdmJlqfRplJnJZupBJxXfMOasgamAixud_1Qo9sEg4kAsopD1Ts

How Sleep Deprivation Affects Psychological Variables Related to College Students' Cognitive Performance. (n.d.). Taylor \& Francis. https://www.tandfonline.com/doi/abs/10.1080/07448489709595597 
Impact of Poor Sleep Quality on the Academic Performance of Medical Students. (2019, April 1). PubMed Central (PMC). https://www.ncbi.nlm.nih.gov/pmc/articles/PMC6550515/

Joo, E. Y., Yoon, C. W., Koo, D. L., Kim, D., \& Hong, S. B. (2012). Adverse effects of 24 hours of sleep deprivation on cognition and stress hormones. Journal of clinical neurology (Seoul, Korea), 8(2), 146. https://pubmed.ncbi.nlm.nih.gov/22787499/

LibGuides: $\quad$ Qualitative $\quad$ Research $\quad$ Methods: $\quad$ Phenomenology. $\quad$ (2020). LibApps. https://guides.library.duq.edu/c.php?g=836228\&p=5972144

Mantua J., \& Simonelli, G. (2019). Sleep duration and cognition: is there an ideal amount?, Sleep, Volume 42, Issue 3, March 2019, zsz010, https://doi.org/10.1093/sleep/zsz010

Miller, S. G. (2016, April 4). How a Sleepless Night Affects Your Ability to Focus. Livescience.Com. https://www.livescience.com/54284-sleep-deprivation-selective-

attention.html?fbclid=IwAR0VuX7tewqFbNLUKcdQzMdjnHjgE49lfurWurT1mNZTPGJkzMYPpLkIBlg

Noland, H. "Adolescents' Sleep Behaviors and Perceptions of Sleep." American School Health Association. https://www.csus.edu/faculty/m/fred.molitor/docs/sleep.pdf

Paruthi, S. (2016). Recommended Amount of Sleep for Pediatric Populations: A Consensus Statement of the American Academy of Sleep Medicine. (2016 Jun 15). Journal of Clinical sleep medicine. https://www.ncbi.nlm.nih.gov/pmc/articles/PMC4877308/

Prevalence of sleep disorders among university students and its impact on academic performance. (n.d.). Taylor \& Francis. Retrieved June 20, 2021, from https://www.tandfonline.com/doi/full/10.1080/02673843.2020.1815550

Procedures of Colaizzi's phenomenological descriptive method (Colaizzi, 1978). Research Gate. https://www.researchgate.net/figure/Procedures-of-Colaizzis-phenomenologicaldescriptive-method-Colaizzi-1978_fig1_274382296

Professional Stress, Sleep Quality and Coping in Post-Graduate Medical Students-Indian Journals. (2013). https://www.indianjournals.com/ijor.aspx?target=ijor:jrmee \& volume=3\&issue=3\&article=004\#cor 001

Roberts, R., Duong, H., MD, MPH, (2014). The Prospective Association between Sleep Deprivation and Depression among Adolescents, Sleep, Volume 37, Issue 2, 1 February 2014, Pages 239-244, https://doi.org/10.5665/sleep.3388

Sleep-deprived. (n.d.). The Merriam-Webster.Com Dictionary. Retrieved June 21, 2021, from https://www.merriamwebster.com/dictionary/sleep-deprived

Sleep deprivation - Better Health Channel. (n.d.). Retrieved June 21, 2021, from https://www.betterhealth.vic.gov.au/health/conditionsandtreatments/sleep-deprivation\#rpl-skiplink https://www.betterhealth.vic.gov.au/health/ConditionsAndTreatments/sleep-deprivation\#bhccontent

Stieg, C. (2020, February 4). Science says waking up to this specific alarm clock sound can give you more energy, reduce grogginess. CNBC. https://www.cnbc.com/2020/02/04/study-wakingup-to-melodic-sounds-increases-energy-compared-to-alarms.html

Suni, E. (2020a, August 14). Sleep Hygiene. Sleep Foundation. https://www.sleepfoundation.org/sleep-hygiene 
Suni, E. (2020, December 11). How Lack of Sleep Impacts Cognitive Performance and Focus. Sleep Foundation. https://www.sleepfoundation.org/sleep-deprivation/lack-of-sleep-and-cognitive-impairment

Suni, E. (2020a, August 14). Stages of Sleep. Sleep Foundation.

https://www.sleepfoundation.org/how-sleep-works/stages-of-sleep

Suni, E. (2020b, September 18). Mental Health and Sleep. Sleep Foundation. https://www.sleepfoundation.org/mental-health

The Structure of Coping. American Sociological https://www.nederlandsautismeregister.n1/assets/Documenten/References\%20Questionnaires/Coping\%20Methods\%20Questionnaire $\% 20$ (Mastery\%20Scale).pdf

Using Colaizzi's method of data analysis to explore the experiences of nurse academics teaching on satellite campuses. (n.d.). RCNi. https://journals.rcni.com/nurse-researcher/usingcolaizzis-method-of-data-analysis-to-explore-the-experiences-of-nurse-academics teaching-onsatellite-campuses-nr.2018.e1516?fbclid=IwAR0HaG9CnsCGMc_4JJcvMrKfqnGMo8DhQkgMr8OzxoQd5iyfNaYJV SvZJTc\#:\%7E:text=Colaizzi’s\%20(1978)\%20method\%20of\%20data\%20analysis\%20is\%20a\%2 0rigorous\%20and,themes\%20and\%20their\%20interwoven\%20relationships

What Impact Does Sleep Have on Mental Health? (n.d.). Verywell Mind.https://www.verywellmind.com/how-sleep-affects-mentalhealth-4783067

What Lack of Sleep Does to Your Mind. (2009b, December 30). WebMD. https://www.webmd.com/sleep-disorders/features/emotionscognitive

Yang, G. (2014, June 6). Sleep promotes branch-specific formation of dendritic spines after learning. Science. https://science.sciencemag.org/content/344/6188/1173

Zeek, M. L., Savoie, M. J., Song, M., Kennemur, L. M., Qian, J., Jungnickel, P. W., \& Westrick, S. C. (2015). Sleep duration and academic performance among student pharmacists. American journal of pharmaceutical education, 79(5). https://pubmed.ncbi.nlm.nih.gov/26396272/ 